\title{
Das neue Zweitmeinungsverfahren
}

\section{Zusammenfassung}

Seit Inkrafttreten des Versorgungsstärkungsgesetzes (GKV-VSG) im Juli 2015 haben alle gesetzlich krankenversicherte Patienten (GKVPatienten) vor bestimmten medizinischen Eingriffen einen Rechtsanspruch auf Einholung einer unabhängigen ärztlichen Zweitmeinung. Die Neuregelung in § 27 b SGB V betrifft GKV-Patienten, „bei denen die Indikation zu einem planbaren Eingriff gestellt wird, bei dem insbesondere im Hinblick auf die zahlenmäßige Entwicklung seiner Durchführung die Gefahr einer Indikationsausweitung nicht auszuschließen ist“. Der mit dieser Regelung verbundene Vorwurf einer nicht medizinisch begründeten Indikationsausweitung geht offensichtlich auf einen Bericht der Studie der Organisation für wirtschaftliche Zusammenarbeit und Entwicklung (OECD) aus dem Jahr 2013 zurück, demzufolge Deutschland Weltmeister bei der Zahl der Krankenhausbehandlungen und Operationen sein soll - insbesondere bei der Durchführung von Hüftgelenks- und Bypass-Operationen, die als sog. "mengenanfällige Eingriffe" definiert werden (OECD Health at a Glance 2013).

Text

\section{Anspruch auf Zweitmeinung bei planbaren mengenanfälligen Eingriffen}

Derzeit (Februar 2016) steht allerdings noch nicht fest, für welche konkreten planbaren Eingriffe der Zweitmeinungsanspruch obligatorisch sein soll, obwohl der Gemeinsame Bundesausschuss (G-BA) nach dem Willen des Gesetzgebers hierzu erstmals bis zum 31.12.2015 nähere Bestimmungen hätte treffen sollen. Auch hätte der G-BA bis zu diesem Zeitpunkt die fachlichen Anforderungen an eine solche Zweitmeinung und die erforderliche Expertise der hinzugezogenen Experten festlegen sollen. Schon im Frühjahr 2015 teilte der G-BA in seiner Stellungnahme zum Gesetzentwurf mit, dass ein sachgerechter Richtlinienbeschluss bis zum 31.12.2015 nicht realisierbar sei, da eine umfangreiche wissenschaftliche Aufbereitung für die Bestimmung der von der Neuregelung betroffenen Eingriffe notwendig sei. Daher stellte er anheim, die Frist bis zum 31.03.2016 zu verlängern und das Datum im Gesetzestext entsprechend abzuändern. Trotz dieses Einwandes hielt der Gesetzgeber an der Frist zum Jahresende 2015 fest.

Konkrete Auswirkungen wird die gesetzliche Neuregelung daher erst haben, wenn der G-BA in seinen Richtlinien den Kanon der seiner Auffassung nach unter die Neuregelung fallenden planbaren und mengenanfälligen Eingriffe definiert hat. Völlig offen ist in diesem Zusammenhang die Frage, ob die Festlegungen des G-BA in seinen Richtlinien der gerichtlichen Überprüfung unterliegen und ob betroffene Ärzte oder einschlägige medizinisch-wissenschaftliche Fachgesellschaften gegen die Entscheidungen des G-BA ggf. gerichtlich zu Felde ziehen werden. Fest steht jedenfalls bereits jetzt, dass die Neuregelungen im
§ 27 b SGB V in ihrer konkreten Ausgestaltung mehrdeutig und auslegungsbedürftig sind und einige strittige Fragen unter Ärzten und Juristen aufwerfen.

\section{Unabhängigkeit der Zweitmeinung}

Nach den Neuregelungen soll der Patient die ärztliche Zweitmeinung grundsätzlich bei den an der vertragsärztlichen Versorgung teilnehmenden Ärzten und Einrichtungen einholen. Allerdings wird der G-BA in seinem - noch ausstehenden - Richtlinienbeschluss bestimmte Kriterien für die Vertragsärzte festlegen, die zur Abgabe der Zweitmeinung berechtigt sein sollen. Hierzu zählen z.B. eine langjährige fachärztliche Tätigkeit in einem Fachgebiet, das für die Indikation zum Eingriff maßgeblich ist, Kenntnisse über den aktuellen Stand der wissenschaftlichen Forschung zur jeweiligen Diagnostik und Therapie einschließlich der Kenntnisse über Therapiealternativen zum empfohlenen Eingriff.

Zudem darf die Zweitmeinung nicht von demselben Arzt oder von derselben Einrichtung (Krankenhaus, MVZ, Berufsausübungs- oder Partnerschaftsgesellschaft) eingeholt werden, in der der Eingriff durchgeführt werden soll. Ansonsten entfällt der gesonderte Leistungsanspruch des Vertragsarztes für die Zweitmeinung. Laut Gesetzesbegründung sollen hierdurch die Unabhängigkeit der Zweitmeinung gestärkt und gleichzeitig falsche finanzielle Anreize vermieden werden. Danach muss der Patient, der sich ggf. gern von dem von inm konsultierten Experten behandeln lassen würde, weil er eine andere Behandlungsempfehlung als sein Kollege gegeben hat, noch einen dritten Arzt aufsuchen, der den Eingriff dann tatsächlich nach den Empfehlungen des Zweitmeinungsarztes durchführt. 


\section{Aufklärungs- bzw. Informationspflicht des behandelnden Arztes}

Mit der Einführung des Zweitmeinungsanspruchs wird allen die Erstindikation stellenden Ärzten in Klinik und Praxis neben den gesetzlichen Aufklärungspflichten eine zusätzliche Aufklärungs- bzw. Informationspflicht aufgebürdet. Nach § 27 b Abs. 5 SGB V hat der Arzt den Versicherten im Falle von planbaren, mengenanfälligen Eingriffen, die der G-BA festgelegt hat, über das Recht zur Einholung einer unabhängigen ärztlichen Zweitmeinung aufzuklären und inn auf die Informationsangebote der Kassenärztlichen Vereinigungen und der Landeskrankenhausgesellschaften über geeignete Leistungserbringer hinzuweisen. Die Aufklärung muss mündlich erfolgen; ergänzend können schriftliche Informationen in Textform zur Verfügung gestellt werden. Diese formalen Vorgaben orientieren sich an den üblichen Formerfordernissen für die ärztlichen Aufklärungspflichten ( $§ 630$ e Abs. 2 S. 1 Nr. 1 BGB). Diese neuen Informationspflichten bestehen nur bei GKV-Patienten und auch nur in den Fällen, in denen es sich um sog. planbare und mengenanfällige Eingriffe handelt, die der G-BA noch festlegen muss. Für alle anderen Eingriffe und bei vollversicherten Privatpatienten gilt das Zweitmeinungsverfahren nicht.

Wann der G-BA die inm vom Gesetzgeber auferlegten Richtlinien beschließen wird, ist derzeit nicht absehbar; mit der Festlegung sog. Planbarer und mengenanfälliger Eingriffe im Sinne der Neuregelung ist jedoch im Laufe des Jahres 2016 zu rechnen. Erst ab diesem Zeitpunkt müssen die neuen Informationspflichten der Ärzte ernst genommen werden. Maßgeblich ist immer nur das mündliche Aufklärungsgespräch zwischen Arzt und Patient. Das haftungsrechtliche Risiko, das aufgrund einer zumindest vom Patienten behaupteten - unterbliebenen Aufklärung über den Zweitmeinungsanspruch besteht, ist derzeit nicht abschätzbar. Daher ist zu Beweiszwecken die schriftliche Dokumentation der erfolgten Information über den Zweitmeinungsanspruch unbedingt empfehlenswert, um spätere haftungsrechtliche Auseinandersetzungen zu vermeiden.

Die ärztliche Information über den Zweitmeinungsanspruch muss zudem rechtzeitig erfolgen. § 27b Abs. 5 Satz 2 SGB V bestimmt, dass der Arzt dafür Sorge zu tragen hat, dass die Aufklärung in der Regel mindestens zehn Tage vor dem vorgesehenen Eingriff erfolgt, am besten früher. In jedem Fall hat die Aufklärung so rechtzeitig zu erfolgen, dass der Versicherte seine Entscheidung über die Einholung einer Zweitmeinung wohlüberlegt treffen kann. Da der aufklärende Arzt faktisch keine Einwirkungsmöglichkeiten auf die Kapazität anderer Arztpraxen und Krankenhäuser hat, dürfte die Einhaltung der zeitlichen Vorgaben schwierig werden, zumal die Einhaltung der Mindestzeiten von 10 Tagen offensichtlich nur eine Richtschnur sein soll (,in der Regel“).

\section{Haftung bei unterbliebener Information über Zweitmeinungsanspruch?}

Die mit dem Zweitmeinungsverfahren einhergehenden neuen Informationspflichten werden zwangsläufig für Rechtsunsicherheit sorgen. Der Umstand, dass der Gesetzesgeber den Begriff „Aufklärung“ verwendet hat, wird in vielen Fällen zu einer nicht beabsichtigten haftungsrechtlichen Problematik führen. Unseres Erachtens darf die Pflicht, den Patienten auf die Möglichkeit der Einholung einer Zweitmeinung hinzuweisen, nicht mit der ärztlichen Eingriffs- und Sicherungsaufklärung über den tatsächlich indizierten Eingriff verwechselt oder gleichgestellt werden. Bei Verstößen gegen die Eingriffs- oder Sicherungsaufklärung können Schadensersatz- und Schmerzensgeldansprüche entstehen. Ein derart weitreichendes Haftungsrisiko infolge einer etwaig unterbliebenen Aufklärung über den Zweitmeinungsanspruch kann vom Gesetzgeber nicht beabsichtigt worden sein. Sinn und Zweck der Neuregelung ist in erster Linie der Rechtsanspruch des Patienten auf Zweitmeinung zur Vermeidung nicht indizierter/unnötiger Operationen. Was aber gilt, wenn der Patient im Falle einer unterbliebenen Information ohne Einholung einer Zweitmeinung operiert wird und sich bei der Operation Komplikationen ergeben? Kann dieser Patient dann den operierenden Arzt auf Schadensersatz und Schmerzensgeld in Anspruch nehmen mit der Begründung, er hätte sich bei Einholung einer Zweitmeinung nicht operieren lassen und die Komplikation wäre dann nicht eingetreten? Wer trägt für ein solches Alternativverhalten die Darlegungs- und Beweislast? Der Arzt, weil er seiner Verpflichtung über die Information zur Zweitmeinung schuldhaft nicht nachgekommen ist? Was gilt, wenn die Informationen über die Möglichkeit der Zweitmeinung zu spät erfolgen und der Patient keine Zeit mehr hat, eine Zweitmeinung einzuholen? Fragen über Fragen, die mit den Neuregelungen verbunden sind. Auf Grund dieser Unwägbarkeiten der Neuregelungen sollte die mündliche Information der Patienten über den Zweitmeinungsanspruch ernst genommen werden; zudem ist eine Dokumentation über die dem Patienten gegenüber abgegebene Information dringend geboten.

\section{Handlungsempfehlung}

Ärzte und medizinische Fachgesellschaften aus Fachgebieten, die voraussichtlich in erster Linie von den Neuregelungen betroffen sein werden (z.B. Herzchirurgen bei Koronarangioplastien - PTCA und bei koronaren BypassOperationen; Orthopäden und Unfallchirurgen bei künstlichen Hüftgelenken und bei Kniegelenkersatz; ggf. auch HNO-Chirurgen bei Tonsillektomien) sollten die Beschlusslage des G-BA über die Festlegung planbarer, mengenanfälliger Eingriffe zum Zweitmeinungsverfahren aufmerksam und zeitnah verfolgen (www.g-ba.de). Zudem ist dringend anzuraten, die bei Zweitmeinungseingriffen erforderliche Aufklärung der Patienten zu dokumentieren und entsprechende Formulare vorzuhalten und sich dazu rechtlich beraten zu lassen. 


\section{Korrespondenzadresse:}

Dr. iur. Albrecht Wienke

Wienke \& Becker - Köln, Sachsenring 6, 50677 Köln Awienke@Kanzlei-WBK.de

\section{Bitte zitieren als}

Wienke A. Das neue Zweitmeinungsverfahren. GMS Mitt AWMF. 2016;13:Doc1.

DOI: 10.3205/awmf000313, URN: urn:nbn:de:0183-awmf0003137
Artikel online frei zugänglich unter

http://www.egms.de/en/journals/awmf/2016-13/awmf000313.shtml

Eingereicht: 11.02.2016

Veröffentlicht: 11.02.2016

\section{Copyright}

(C)2016 Wienke. Dieser Artikel ist ein Open-Access-Artikel und steht unter den Lizenzbedingungen der Creative Commons Attribution 4.0 License (Namensnennung). Lizenz-Angaben siehe http://creativecommons.org/licenses/by/4.0/. 